\title{
A Search for Rotational Modulation in the EUV Emission from AB Doradus
}

\author{
S. M. WHITE, ${ }^{1}$ J. LIM, ${ }^{2}$ S. M. RUCINSKI, ${ }^{3}$ G. ROBERTS, ${ }^{4}$ \\ D. KILKENNY, ${ }^{4}$ S. G. RYAN, ${ }^{5}$ P. PRADO,${ }^{6}$ AND M. R. KUNDU ${ }^{1}$ \\ ${ }^{1}$ Dept. of Astronomy, Univ, of Maryland, College Park MD 20742, USA \\ ${ }^{2}$ IAA, Academica Sinica, PO Box 1-87, Nankang, Taipei, Taiwan \\ ${ }^{3}$ Inst. for Space and Terr. Science, and York Univ., 4850 Keele St., Toronto, \\ Ontario M3J 3K1, Canada \\ ${ }^{4}$ South African Astronomical Observatory, PO Box 9, Observatory 7935, South Africa \\ ${ }^{5}$ Anglo-Australian Observatory, PO Box 296, Epping NSW 2121, UK \\ ${ }^{6}$ Las Campanas Observatory Casilla 601, La Serena, Chile
}

One of the goals of the $E U V E$ observation of $\mathrm{AB}$ Doradus was to search for rotational modulation of the EUV emissions. In support of this goal we carried out optical photometry in Chile, Australia and South Africa and radio observations in Australia. In addition, an $A S C A$ observation of AB Dor was scheduled to occur during the campaign. Several spectacular X-ray and optical flares with accompanying brightening in the EUV were seen, but no rotational modulation of the EUV emission was evident, except for a dip in the He II $304 \AA$ line coincident with the optical minimum. An X-ray flare was seen with no accompanying EUV flare.

\section{Introduction}

AB Doradus is a very active nearby southern $K$ dwarf star. This object has attracted attention for a number of reasons. It is one of the most rapidly-rotating single stars known, with a period of only 12.4 hours, and it is relatively close, at $25 \mathrm{pc}$. Its rotation axis is inclined at $60^{\circ}$ to our line of sight. Further, it is a young star: it has a strong $\mathrm{Li}$ absorption line (Rucinski 1985), and kinematic properties characteristic of the Pleiades group (Innis, Thompson, \& Coates 1986). This makes it probably the nearest pre-main-sequence star known, with an age of approximately 50 million years; its closeness makes it an easily-studied analogue of the numerous rapidlyrotating dwarf stars in the Pleiades open cluster. Prior X-ray observations by EINSTEIN (Pakull 1981), EXOSAT (Cameron et al. 1988), GINGA (Vilhu et al. 1993) and ROSAT (Kürster, Schmitt, \& Fleming 1992) revealed AB Dor to be a strong and interesting coronal source. In addition, radio observations have shown a remarkable modulation of the light curve at the rotation period ( $\mathrm{Lim}$ et al. 1992) which can be used to analyze the distribution of radio-emitting material in the stellar atmosphere (Lim et al. 1994).

EUVE observed AB Dor from 1993 Nov 4.4-11.2 (total exposure of $140 \mathrm{ks}$ ). Rucinski et al. (1995 and this volume) present a detailed analysis of the EUV spectrum of AB Dor; in this paper we focus on a search for rotational modulation in the photometric data and on the results of a coordinated campaign of supporting observations. This included U-band photometry at the South African Astronomical Observatory SAAO), with 2 s time resolution, on Nov 4-8; U-band CCD photometry at the U. Toronto Las Campanas facility on Nov 4, 5, \& 7; and U-band CCD photometry at Mount Stromlo Siding Spring Observatory (MSSSO) on Nov 5-7. CCD photometry was used at the latter two sites to establish the relevance of AB Dor's M dwarf companion, Rst $137 \mathrm{~B}$, which is $9^{\prime \prime}$ away and therefore is not resolved by EUVE. Rst 137B showed several very impulsive optical flares but should not be contributing in any of the data shown. Both optically and in 


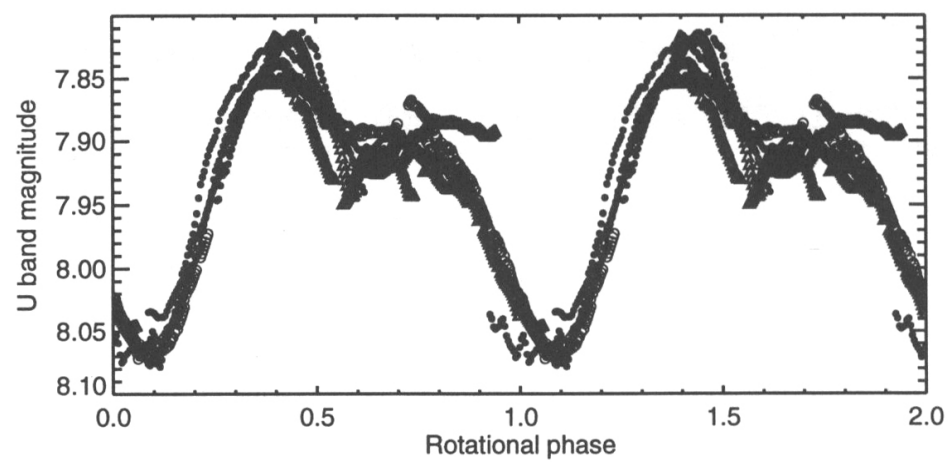

Figure 1. Optical U-band data taken during the campaign plotted versus rotational phase using the ephemeris of Innis et al. (1988). The largest optical flare has beem removed from the data.

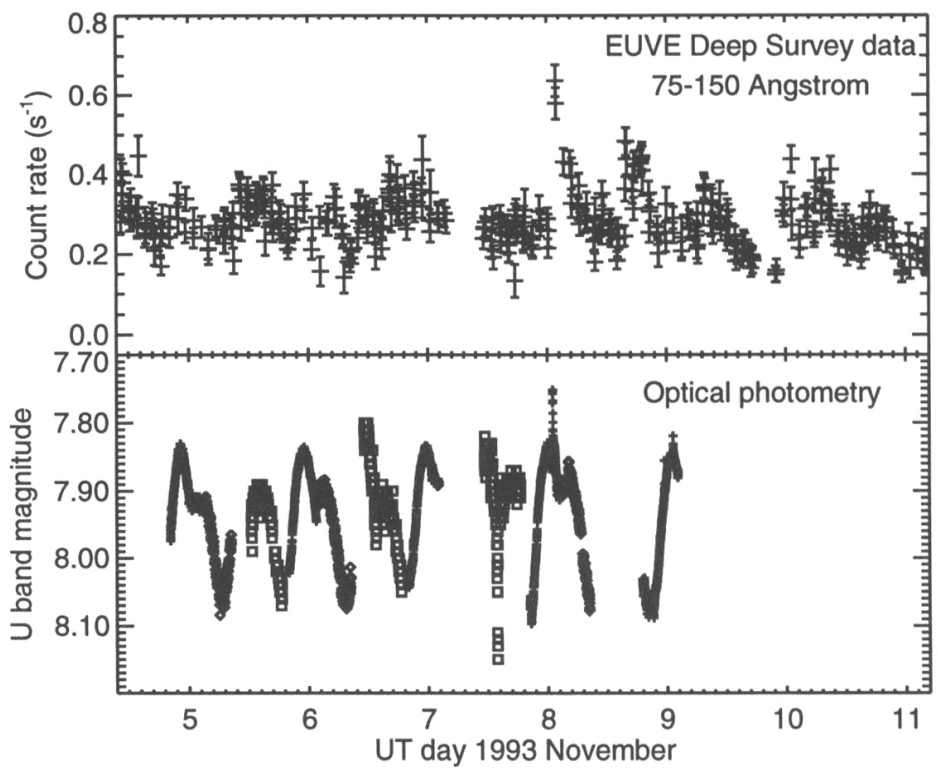

FIGURE 2. The light curve of the EUVE DS data (upper panel) and the U-band optical light curve (lower panel) for the duration of the EUVE observation.

$\mathrm{X}$-rays it is a factor of 60 fainter than AB Dor. We obtained radio observations at 1.4, 2.4, 5.0, \& 8.0 GHz with the Australia Telescope on November 5 and 6 . In addition the Japanese X-ray satellite $A S C A$ observed AB Dor from Nov 7.55-8.25 (total exposure of $40 \mathrm{ks}$ ). 


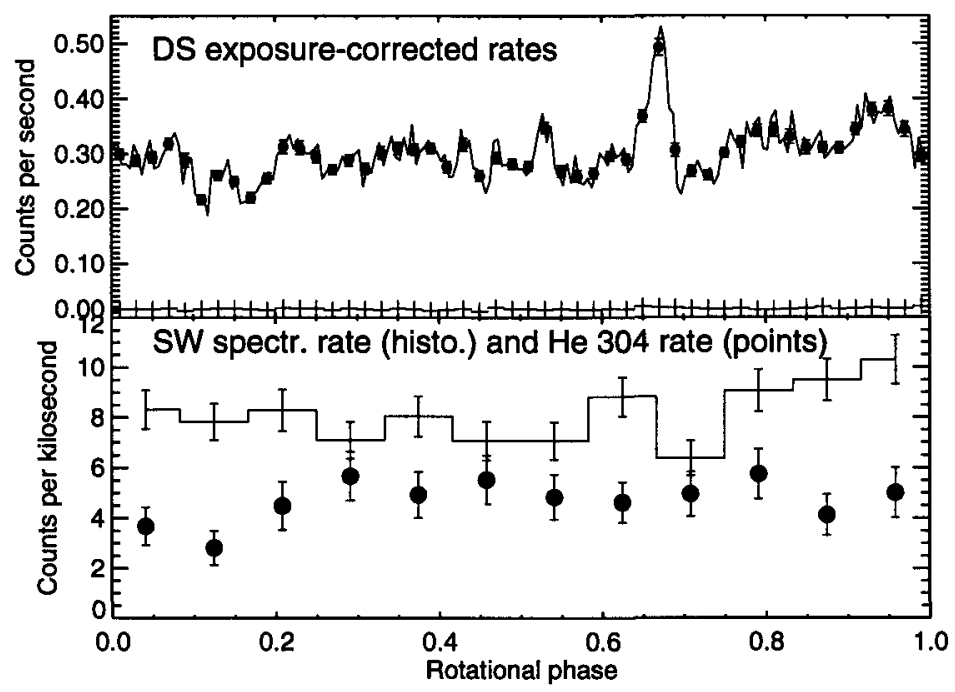

FIGURE 3. The EUVE DS data plotted against rotational phase (upper panel). The points with error bars correspond to 50 bins per orbit, while the solid line corresponds to 200 bins per orbit. The crosses at the bottom of the upper panel show the background count rate. In the lower panel we plot the count rate in the SW spectrometer (histogram with $1 \sigma$ error bars), which is a diagnostic of the hot plasma in the corona similar to the DS data, and the counts in the strong He II $304 \AA$ line (points), which is a transition-region diagnostic.

\section{Observational Data}

Figure 1 shows all the photometry data plotted against rotational phase using the ephemeris of Innis et al. (1986). In Figure 1, filled circles represent SAAO data, binned to 2 minute resolution; open circles represent Las Campanas data; and open triangles represent MSSSO data. While the intrinsic scatter in the data is less than 0.01 magnitudes, the absolute level is not well determined at the three observatories, and relative shifts of up to 0.1 magnitudes were used to line up the light curves. The data show a clear modulation of 0.2 magnitudes in $U$. The star is brightest at a phase of 0.4 , and faintest at 0.1. AB Dor's light curve has been monitored for over a decade and this is the largest level of variability seen in the light curve in that time.

In Figure 2 we show the EUV light curve using data from the Deep Survey (DS) imager, which is the most sensitive detector for EUV photometry. Its passband corresponds to that of the SW spectrometer. In this figure we have binned the photons into $400 \mathrm{~s}$ bins. Due to AB Dor's high ecliptic latitude the observing efficiency was only $24 \%$. Several weak flares may be seen. In Figure 3 we have divided the rotation period into a number of bins and allocated the DS data according to rotational phase. The top panel shows the count rate as a function of phase, while the bottom panel shows two diagnostics from spectrometer data: total counts in the 80-170 $\AA$ range in the SW detector; and counts in the He II $304 \AA$ line (MW and LW combined). Unlike the optical data, the DS data show no striking modulation with rotational period. The narrow feature in the phase-binned DS data at phase 0.67 is due to the combination of a low exposure time in that particular bin, and the coincidence of having several flares contribute to the bin.

Figure 4 shows the $A S C A$ count rate, the $E U V E$ DS data and the optical photometry 


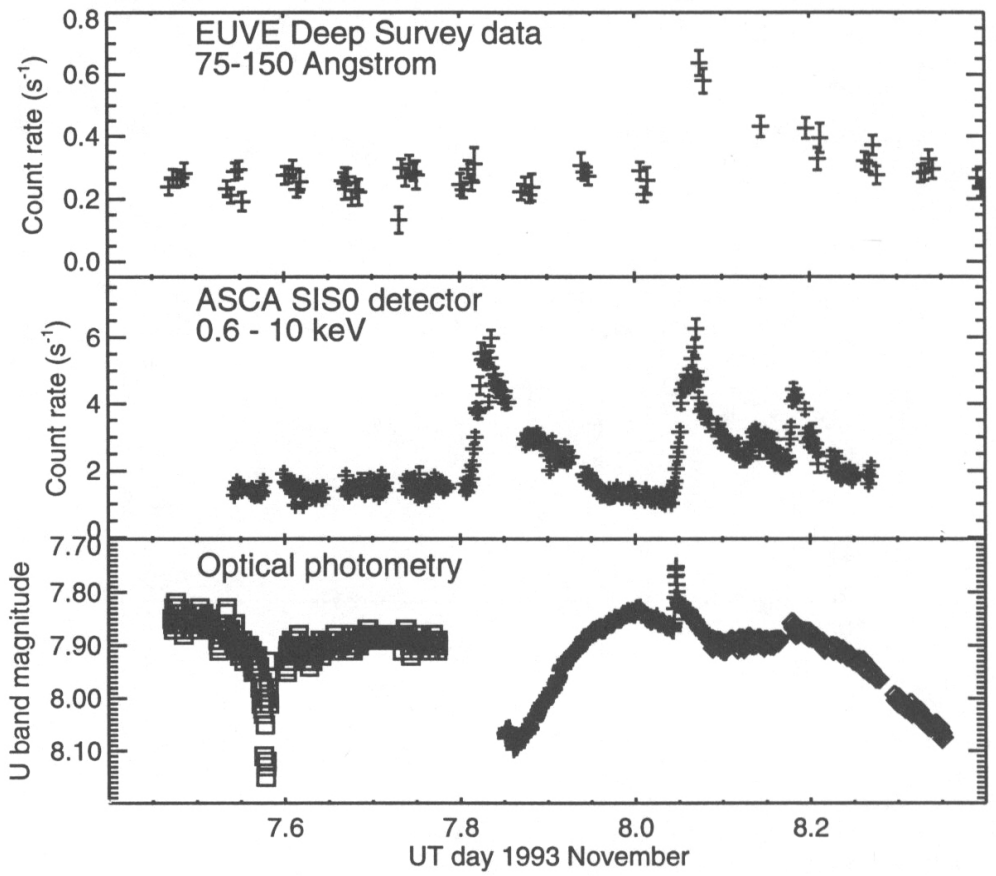

FIGURE 4. The middle panel shows the count rate in the ASCA SIS0 detector (0.6-10 keV) during the $A S C A$ observation of $\mathrm{AB}$ Dor on Nov 7-8. The upper panel shows the light curve of the EUVE DS data and the lower panel the optical light curve during the same period.

during the period of the $A S C A$ observation. The $A S C A$ data show a very steady level of just under 2 counts $\mathrm{s}^{-1}$ for the first $6 \mathrm{hrs}$ of the observation, but the remaining period is dominated by a succession of flares reaching up to 6 counts $\mathrm{s}^{-1}$. Two of the $\mathrm{X}$-ray flares (at Nov 8.05 and 8.17) are coincident with optical flares. The $E U V E$ light curve is unchanged following the first major flare at Nov 7.8, but at least doubles following the second major X-ray flare and shows a gradual decay over the following 6 hours. Since the two large flares occur about $6 \mathrm{hrs}$ apart, then they either come from the always-visible region near the pole, or else they come from opposite hemispheres of the star. The second large flare is coincident with a large optical flare, as is one of the small later events.

\section{Discussion}

The main conclusion of the data collected during the EUVE AB Dor observation is that there was little evidence for rotational modulation of the coronal diagnostics (EUV, radio and $\mathrm{X}$-ray). This result can be interpreted as either an approximately uniform distribution of material with longitude on the visible hemisphere, or else a very extended corona in which most of the coronal material is visible at all times. Unlike the radio data (Lim et al. 1992, Lim et al. 1994), the previous EUV (Pagano et al. 1993) and X-ray observations (Cameron et al. 1988, Vilhu et al. 1993, Kürster, Schmitt, \& Fleming 1992) have also found little compelling evidence for rotational modulation. 
However, there is evidence for modulation of the transition-region temperature diagnostic, the He II $304 \AA$ line, which appears to have a minimum at the minimum in the optical light curve. This suggests weak transition-region emission above a large spot at the minimum of the optical light curve.

We believe that AB Dor was in a low-activity state until about Nov 7.8. Three pieces of evidence suggest this: although about two-thirds of the optical photometry took place prior to this time, no optical flares were detected prior to this time, but four optical flares were detected following it; no EUV flares were detected prior to this time, whereas at least 2 were detected subsequently; and the $\mathrm{X}$-ray light curve is very steady until this time, but shows a succession of flares thereafter. The radio data (not shown) were taken prior to this time and show clear variability, but no unambiguous rotational modulation. The relatively low level of radio emission (a few $\mathrm{mJy}$ at all frequencies) is consistent with the discussion by Lim et al. (1994), who noted that the radio modulation is most pronounced when the radio flux is high (tens of $\mathrm{mJy}$ ).

It is a puzzle to understand why one large $\mathrm{X}$-ray flare should produce an obvious response in SW EUV emission when another of similar size did not. The EUV emission in the DS detector is dominated by lines of highly-ionized Fe, and we expect it to respond to the same (in this case, 10-30 MK) plasma which produces the X-rays, as it did in the second flare.

SMW gratefully acknowledges support from NASA's $E U V E$ and $A S C A$ Guest Investigator programs (grants NAG-5-2364 and NAG-5-2531, respectively), and from NSF grant AST 92-17891. SMR's research has been supported by a grant from the Natural Sciences and Engineering Research Council of Canada.

\section{REFERENCES}

Cameron, A. C., Bedford, D. K., Rucinski, S. M., Vihhu, O., \& White, N. E. 1988, MNRAS, 231, 131

Innis, J. L., Thompson, K., \& CoAtes, D. W. 1986, MNRAS, 223, 183

Kürster, M., Schmitt, J. H. M. M., \& Fleming, T. A. 1992, in Cool Stars, Stellar Systems and the Sun, 7th Cambridge Workshop, ed. M. S. Giampapa \& J. A. Bookbinder, San Francisco: ASP Conf. Ser. 26, 109

Lm, J., Nelson, G., Castro, C., Kilkenny, D., \& van WyK, F. 1992, ApJL, 388, 27

LIM, J., White, S. M., Nelson, G. J., \& BenZ, A. O. 1994, ApJ, 430, 332

Pagano, I., ET AL. 1993, in Physics of Solar and Stellar Coronae, ed. J. F. Linsky \& S. Serio Dordrecht: Kluwer Academic Press, 457

PAKULl, M. W. 1981, A\&A, 104, 33

RUCINSKI, S. M. 1985, MNRAS, 215, 591

Rucinski, S. M., Mewe, R., KaAstra, J. S., Vilhu, O., \& White, S. M. 1995, ApJ, in press

Vilhu, O., Tsuru, T., Collier, Cameron, A., Budding, E., Banks, T., Slee, B., EhrenFREUND, P., \& ForNG, B. H. 1993, A\&A, 278, 467 